\title{
The Effect of Micro-Pulsatile Electrical and Ultrasound Stimulation on Cellular Biosynthetic Activities Such as Cellular Proliferation, Endogenous Nitrogen Oxide and Collagen Synthesis
}

\author{
Doris-Antoinette Mbeh, Insaf Hadjab, Mihaela-Elena Ungur, L'Hocine Yahia \\ Laboratory of Innovation and Analysis of Bioperformance (LIAB), Mechanical/Biomedical Engineering \\ Department, Polytechnique Montréal, Montreal, Canada \\ Email: doris-antoinette.mbeh@polymtl.ca, hadjab.insaf@gmail.com, Ihocine.yahia@polymtl.ca, \\ mungur@silhouettone.com
}

Received 18 February 2016; accepted 19 March 2016; published 22 March 2016

Copyright (C) 2016 by authors and Scientific Research Publishing Inc.

This work is licensed under the Creative Commons Attribution International License (CC BY).

http://creativecommons.org/licenses/by/4.0/

(c) (i) Open Access

\section{Abstract}

The skin barrier poses an ongoing challenge for the cosmetics industry. Its penetration, by noninvasive means, can readily be achieved with currents and ultrasound or radiofrequency devices through electroporation, sonophoresis, iontophoresis or cavitation. When several types of energy are applied simultaneously, we expect the effects to be magnified and all the more effective. Although the mechanism of action of each technology on the skin is not entirely controlled, and is even less so when multiple technologies are applied concurrently, some studies demonstrate that nitric oxide (NO) plays a pivotal role in skin wound-healing and regeneration. With regard to wound healing, one of the key functions of NO appears to be its permissive effect on keratinocyte and fibroblast proliferation, which helps promote wound re-epithelialization. The objective of the actual research is to gain an in-depth understanding of the mechanisms generated by NO through the application of a specific combination of technologies.

\section{Keywords}

Collagen, Fibroblasts, NO, Aesthetic Technologies, Ultrasounds, Electric Current 


\section{Introduction}

Throughout the course of the $21^{\text {st }}$ century, the average age of the population is expected to continue to rise, especially in industrialized countries. Increased wrinkling, sagging skin, and laxity are the most visible consequences of human aging [1]. People invest much time and money in rejuvenation procedures, of which many lack efficacy [2].

Aging of the skin is induced by both intrinsic, and extrinsic factors [3] [4], all leading to reduced structural integrity and loss of physiological function [4].

In the skin, about $1.5 \%$ - $5 \%$ of the consumed oxygen is converted into reactive oxygen species (ROS) by intrinsic processes [10]. ROS are continuously produced in the mitochondria as by-products of aerobic metabolic processes through the electron transport chain, and are regarded as the main cause of intrinsic aging [3]. Keratinocytes and fibroblasts are the main producers of "mitochondrial” ROS in the skin. Two other key events associated with intrinsic skin aging are a decrease in cell replication and further degradation of the extracellular matrix. The replicative ability of all dividing cells decreases with time [5]. This process is called cellular senescence. Most visible skin damage occurs in the connective tissue, also referred to as the dermal extracellular matrix (ECM), collagen, the main component of the dermal ECM in mammals, and produced essentially by fibroblasts, is the major determinant of the skin's appearance and also provides tensile strength and stability to the dermal tissue and blood vessels [6].

Extrinsic aging is caused by environmental oxidative factors, such as solar radiation [7], cigarette smoke [8] and other pollutants. An epidemiological study revealed that long-term contact with tobacco smoke and UVAexposure both independently caused accelerated skin aging [9].

In both intrinsic and extrinsic aging, qualitative and quantitative changes in these structures are observed. This leads to loss of tensile strength and recoil capacity, wrinkle formation, dryness, impaired wound healing and increased fragility [10].

Several therapies, including physical forces, have been explored for their ability to enhance cell and tissue regeneration. Among these physical forces are therapeutic ultrasound [11], iontophoresis [12] and electroporation [13].

Therapeutic ultrasound has been used extensively to treat a variety of conditions because of its documented thermal effects [14]. It has repeatedly been shown to increase tissue temperature at depths up to $5 \mathrm{~cm}$ with only minimal increases in skin temperature [11]. It has been suggested that an increase of $1^{\circ} \mathrm{C}$ (mild heating) over baseline muscle temperature of $36^{\circ} \mathrm{C}$ to $37^{\circ} \mathrm{C}$ accelerates the metabolic rate in tissue. An increase of $2^{\circ} \mathrm{C}$ to $3^{\circ} \mathrm{C}$ (moderate heating) reduces muscle spasm, pain, and chronic inflammation and increases blood flow [15]. Vigorous heating, or an increase of $4^{\circ} \mathrm{C}$ or more, has been suggested to alter the viscoelastic properties of collagen and inhibit sympathetic activity [16].

Iontophoresis is the non-invasive process of driving ionized drugs or chemicals into the skin by means of an applied electric field generated by low direct current (DC), continuous (galvanic) or pulsed (microcurrents) [12].

Certain electrical fields, when applied across a cell, have the ability to permeabilize the cell membrane through a process termed "electroporation" in the early 1980s [17]. The mechanism through which the cell membrane is permeabilized is not yet fully understood. It is thought to be related to the formation of nano-scale pores in the cell membrane, from which the term "poration" was derived. Some electrical fields permeabilize the cell membrane temporarily, after which the cells survive ("reversible electroporation"). Other fields can cause the cell membrane to become permanently permeabilized, after which the cells die ("irreversible electroporation"). Reversible electroporation has become an important tool in biotechnology and medicine, especially in the permeabilization of the cell to molecules that normally do not penetrate the membrane, such as genes or drugs.

\section{Materials and Methods}

\subsection{Design}

In this present study, the combination of five scientifically-proven technologies, namely ultrasound (US), iontophoresis currents-also known as impulse microcurrents (MC), electroporation (ELPO) and high-voltage pulsatile currents (HVPC), were applied on fibroblast cells to learn more about the individual and synergic effects on cell proliferation and collagen synthesis. A safety assessment was conducted simultaneously. In addition, we were also seeking to establish a correlation between different stimuli, the production of nitric oxide (NO), and the production of collagen and cell proliferation. Nitric oxide (NO) has been suggested to be a pathophysiological 
modulator of cell proliferation, cell cycle arrest, and apoptosis. Several studies have indicated that low relative concentrations of NO seem to favor cell proliferation and anti-apoptotic responses and higher levels of NO favor pathways inducing cell cycle arrest, mitochondria respiration, senescence, or apoptosis [18].

The individual stimulations were: ultrasound at a frequency of $3 \mathrm{MHz}$, microcurrents with an intensity level of 500 and $600 \mu \mathrm{A}$, electroporation, high-voltage pulsatile currents, control (no stimulation) and basic (placebo $=$ stimulation environment).

The combined stimulations were: Zone I and Zone II stimulations as shown in Table 1.

\subsection{Equipment}

Please see Figure 1 below: The medical device RMD: STRUCTURAL REMODELING SYSTEM, conceived and manufactured by ST. Medic, a division of Silhouet-Tone, Canada.

\subsection{Cell Culture}

Murine fibroblast-derived cell line L929 (American Type Culture Collection, NCTC clone 929 of strain L) was used for the experiments. Cells were cultured in Eagle's Minimum Essential Medium (EMEM) supplemented with $10 \%$ fetal bovine serum (FBS) and 1\% penicillin/streptomycin, and maintained in $25 \mathrm{~cm}^{2}$ polystyrene culture flasks at $37^{\circ} \mathrm{C}$ in a humidified atmosphere of $5 / 95 \% \mathrm{CO}_{2} /$ air. Subcultures were obtained after reaching $70 \%$ - $80 \%$ confluence by trypsinization ( $0.25 \%$ trypsin in citrate saline). In order to test the cytotoxicity of the materials, cell suspensions were plated in a 12 well-plate in a density of $1 \times 10^{5}$ cells/well with EMEM supplemented with $10 \%$ FBS and kept overnight at $37^{\circ} \mathrm{C}$ in a $5 \% \mathrm{CO}_{2}$ humidified atmosphere. Then, each well was treated with different stimulations for $5 \mathrm{~min}$. After $24 \mathrm{~h}$ of incubation, viability was measured using the trypan blue method according to the manufacturer's protocol. Untreated cells were used as a negative control (C-) for normalization. Experiments were replicated three times.

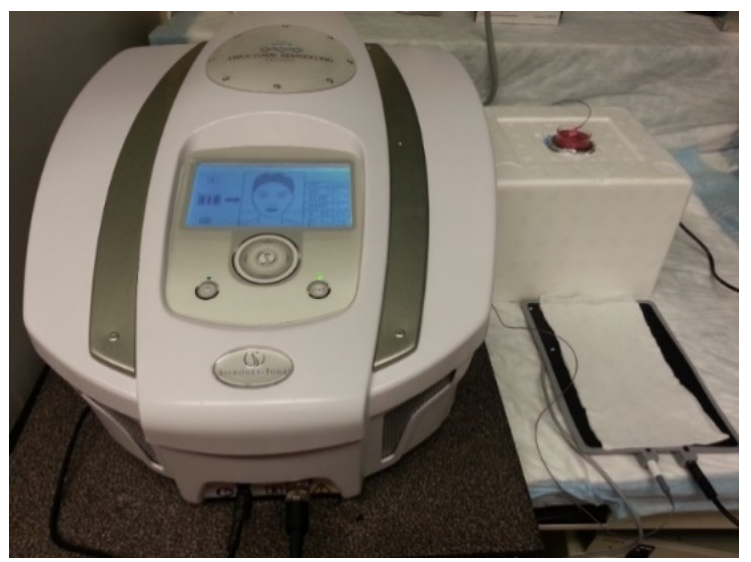

Figure 1. A stimulation device.

Table 1. Individual stimulation and combinations.

\begin{tabular}{ccccc}
\hline Types of stimulations & US & MC & ELPO & 0 \\
\hline Control & 0 & 0 & 0 & 0 \\
Basic & 0 & $100 \mu \mathrm{A}$ & 0 & 0 \\
Ultrasound & $50 \%$ and $75 \%$ & $100 \mu \mathrm{A}$ & 0 & 0 \\
Microcurrents & 0 & 500 and $600 \mu \mathrm{A}$ & $\mathrm{T} 1$ & 0 \\
Electroporation & 0 & $100 \mu \mathrm{A}$ & 0 & 0 \\
HVPC & 0 & $100 \mu \mathrm{A} 1$ \\
Zone I & $50 \%$ & $500 \mu \mathrm{A}$ & 0 & $\mathrm{~T} 1$ \\
Zone II & $75 \%$ & $600 \mu \mathrm{A}$ & $\mathrm{T} 1$ \\
\hline
\end{tabular}




\subsection{Assessment of Cellular Morphology by Microscopy}

In order to test the morphology of the cells after the different stimulations, L929 cells $\left(1 \times 10^{5}\right.$ cells/well $)$ were cultured in a single well plate of $35 \mathrm{~mm}$ for $24 \mathrm{~h}$. Each plate received a different stimulation for 5 minutes and was incubated at $37^{\circ} \mathrm{C}$ and $5 \% \mathrm{CO}_{2}$ continued for another $24 \mathrm{~h}$. Finally, each plate was properly mounted in a microscope. All images were collected using the Leica TCS SP5 inverted Laser Scanning Confocal Microscope (LSCM) with the DM600 fixed stage microscope and the Leica Application Suite software. The settings for the Laser Scanning Confocal Microscope were originally chosen as outlined by the manufacturer's description.

\subsection{Determination of Cell Viability by Trypan Blue Vital Dye Exclusion Assay}

To determine the number of cells and their viability using trypan blue, $100 \mathrm{ml}$ of trypsinized and re-suspended cells were mixed with $20 \mathrm{ml}$ of $0.4 \%$ trypan blue dye solution (Sigma-Aldrich) for $1 \mathrm{~min}$. Cells were immediately counted using a Neubauermicrochamber with a light microscope. All counts were done using four technical duplicates of each sample.

\subsection{Endogenous Nitric Oxide Quantification Using Griess Assay}

The free radical nature of NO makes it highly reactive and very short-lived in biological systems. Therefore, NO is generally viewed as an autocrine or paracrine messenger, mostly regulating local intracellular processes or acting on cells in its near vicinity. The major metabolic pathway for NO involves its rapid oxidation into the higher nitrogen oxides nitrite and nitrate. While the colorimetric Griess reagent detects nitrite, nitrate needs to be reduced to nitrite for detection. NO produced by fibroblast cells after stimulation was estimated spectrophotometrically as a formed nitrite $\left(\mathrm{NO}_{2}\right)$. $\mathrm{NO}$ synthesized by fibroblasts dissolves in medium and is then oxidized to yield both nitrate and nitrite. Twenty four hours after stimulation, $500 \mathrm{ml}$ of culture medium was taken from each well. To measure the nitrite content, $100 \mathrm{ml}$ of the culture medium was incubated with $100 \mathrm{ml}$ of Griess reagent ( $1 \%$ sulfanilamide in $0.1 \mathrm{~mol} / \mathrm{HCl}$ and $0.1 \% \mathrm{~N}$-(1-naphthyl) ethylenediamine dihydrochloride) at room temperature for $10 \mathrm{~min}$. Then the absorbance was measured at $540 \mathrm{~nm}$ using a microplate reader. The nitrite content was calculated based on a standard curve constructed with $\mathrm{NaNO}_{2}$.

\subsection{Collagen Synthesis by Sircol Soluble Collagen Assay}

The effects of stimulation on fibroblast collagen synthesis were determined using a collagen assay test kit (Sircol, Biocolor Ltd.). EMEM media, in the absence of a sample, was maintained as negative control. As per the Sircol instructions, the supernatant was collected and mixed with the Sircol dye reagent and gently mixed at room temperature for $30 \mathrm{~min}$, followed by centrifugation at $1500 \mathrm{~g}$ for $10 \mathrm{~min}$ to collect the collagen-dye pellets. The samples were subsequently dissolved in $750 \mu \mathrm{L}$ alkali reagent and absorbance was measured at $555 \mathrm{~nm}$. The data was expressed in terms of optical density of collagen absorbance by spectrophotometer.

\section{Results}

\subsection{Microscopy}

Our results show that cell density increases after stimulation. The different stimuli do not exhibit the same cellular proliferation. The largest cell densities occur upon stimulation with ultrasound (50\%), microcurrent (500 $\mu \mathrm{A}$ ) and the zone II combination (Figure 2).

\subsection{Cell Proliferation Increases with Stimulation}

Trypan blue staining analysis revealed that cell proliferation increases with stimulation. Aside from electroporation, all other forms of stimulation cause an increase in the number of living cells 24 hours after stimulation compared to control. Stimulation known as Zone II, which is the combination of all stimuli (ultrasound, microcurrent, electroporation and HVPC), has the highest proliferation rate compared to zone I, which is a combination of two types of stimuli (US and MC) (Figure 3). Microcurrents appear to stimulate proliferation more than ultrasound. 
(a) Control

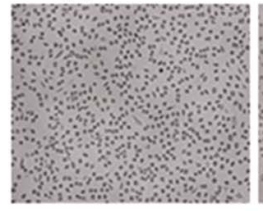

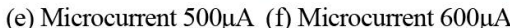

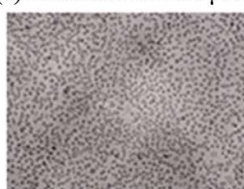

(b) Basic
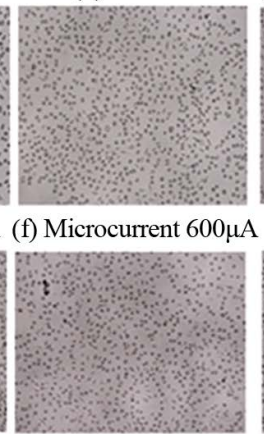

(i) Zone 1

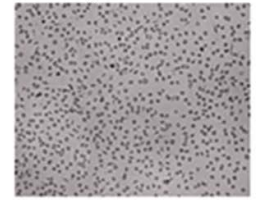

(c) Ultrasound 50\%

(d) Ultrasound $75 \%$

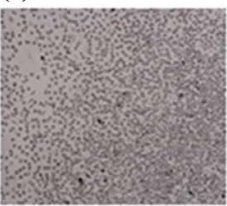

(g) ELPO T 1

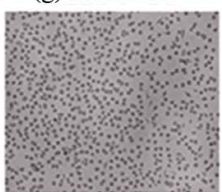

(j) Zone 2

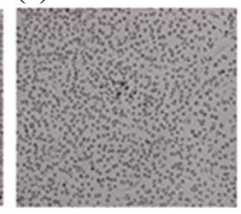

(h) HVPC T1

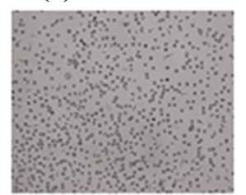

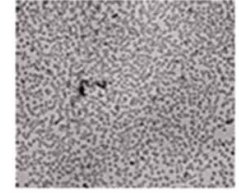

Figure 2. The distribution of fibroblast cells $24 \mathrm{~h}$ after the different stimulations by microscope analysis.

Cell viability

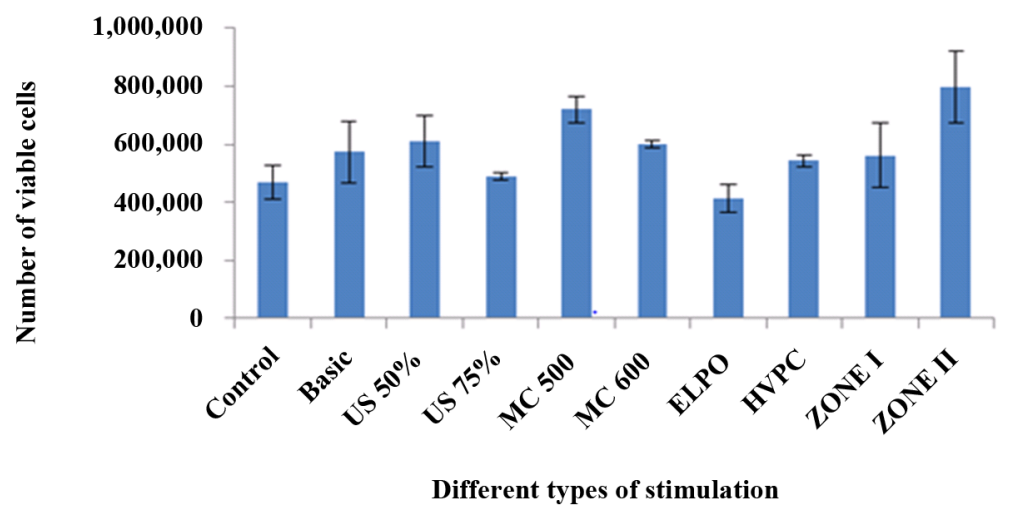

Figure 3. Effect of 5 minutes of stimulation to ultrasound, microcurrent, iontophoresis and electroporation on cell viability of L929 fibroblasts after 24 hours of incubation. (Control: unstimulated cells, basic = placebo: cells exposed to the conditions of stimulation but not stimulated). Values represent the mean \pm standard error of the mean (SEM) ( $n=4$ independent assays), and each experiment was performed in triplicate.

\subsection{Stimulation Boosts the Production of Nitric Oxide}

Stimulation has an immediate effect on the production of nitric oxide (NO): The Control and Basic conditions show no nitric oxide before $24 \mathrm{~h}$. Stimulation by ultrasound shows the synthesis of nitrogen monoxide 1 hour after, while the other stimulations show nitric oxide just 5 min after stimulation. As shown in Figure 4, at the cellular level, 24 hours after stimulation, individual stimuli are similar to the Basic condition. However, the synergy of the five technologies, represented by Zone II, shows a greater production of nitric oxide. The amount of nitric oxide increases over time. HVPC presents the maximum production of nitric oxide 5 minutes after stimulation, while it is after 20 minutes for zone I. The production of nitric oxide for Zone II is higher than others at 35 min.

\subsection{Enhancement of Fibroblast Collagenesis}

Collagen is a protein in the extracellular matrix that is synthesized by fibroblasts in the dermis, where the 


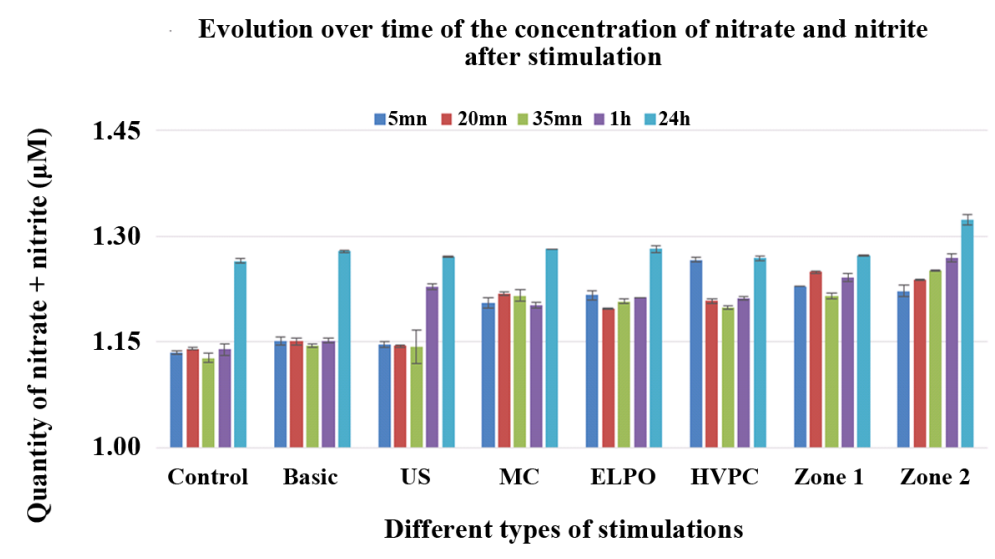

Figure 4. Concentrations of nitrate/nitrite in cell culture supernatants after stimulation. Experiments were performed in triplicate. Values represent the mean \pm standard error of the mean (SEM) ( $\mathrm{n}=3$ independent assays).

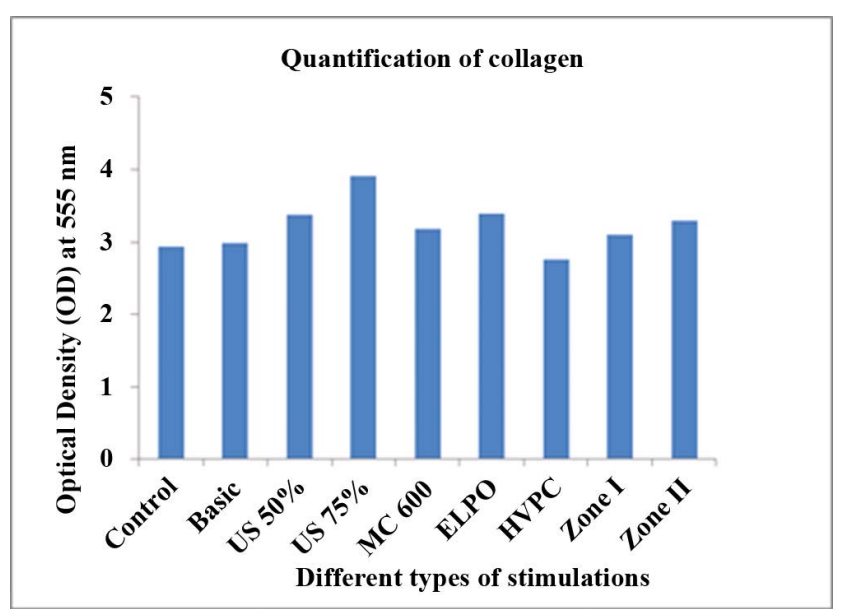

Figure 5. Effect of different stimulations on fibroblast collagen synthesis.

balance between collagen synthesis and degradation is an important element in the wound healing and skin rejuvenation processes. With regard to the wound healing effects of the KRB extract one day and two days posttreatment, our results show that collagen synthesis takes place after individual stimulations such as ultrasound, electrophoresis and also after combined stimuli are applied. However, HVPC does not induce collagenesis, as shown in Figure 5.

\section{Conclusions}

In conclusion, our data support the hypothesis that the combination of ultrasound, microcurrent, electroporation and iontophoresis stimulation is more beneficial than any individual application. The stimulations demonstrated the enhancement of fibroblast proliferation as well as nitric oxide and collagen synthesis. Furthermore, knowing just how important a role cell regeneration, nitric oxide and collagen synthesis play in wound healing and antiaging and, based on the mechanisms of action involved, the benefits of these synergic applications on cell regeneration, hence wound healing and skin aging, is evidenced.

Our research was focused on cellular biosynthetic activities under the effect of micro-pulsatile electrical and ultrasound stimulation. The main limitation of this study was to investigate the response of the cells to the stimulation, without taking in consideration the extracellular matrix (ECM) which may have an additional effect on the cellular response. Therefore, it would be interesting to repeat these experiments in an environment mimicking the physiologic one by ensuring the presence of natural and/or synthetic ECM, constant controlled temperature, and hydrated state. 
Despite the beneficial effects of the combination of aforementioned technologies on the stimulation of fibroblasts and NO synthesis, further studies are required in order to evaluate the relationship between NO synthesis, the multiplication of fibroblasts and collagen synthesis and the involvement of these mechanisms in skin aging and other applications.

\section{References}

[1] Jenkins, G. (2002) Molecular Mechanisms of Skin Ageing. Mechanism of Ageing Development, 123, 801-810. http://dx.doi.org/10.1016/S0047-6374(01)00425-0

[2] Zouboulis, C.C. and Makrantonaki, E. (2011) Clinical Aspects and Molecular Diagnostics of Skin Aging. Clinics in Dermatology, 29, 3-14. http://dx.doi.org/10.1016/j.clindermatol.2010.07.001

[3] Farage, M.A., Miller, K.W., Elsner, P. and Maibach, H.I. (2008) Intrinsic and Extrinsic Factors in Skin Ageing: A Review. International Journal of Cosmetic Science, 30, 87-95. http://dx.doi.org/10.1111/j.1468-2494.2007.00415.X

[4] Landau, M. (2007) Exogenous Factors in Skin Aging. Current Problems in Dermatology, 35, 1-13. http://dx.doi.org/10.1159/000106405

[5] Dimri, G.P., Lee, X., Basile, G., Acosta, M., Scott, G., Roskelley, C., Medrano, E.E., Linskens, M., Rubelj, I. and Pereira-Smith, O. (1995) A Biomarker That Identifies Senescent Human Cells in Culture and in Aging Skin in Vivo. Proceedings of the National Academy of Sciences of the United States of America, 92, 9363-9367. http://dx.doi.org/10.1073/pnas.92.20.9363

[6] Oxlund, H. and Andreassen, T.T. (1980) The Roles of Hyaluronic Acid, Collagen and Elastin in the Mechanical Properties of Connective Tissues. Journal of Anatomy, 131, 611-620.

[7] Wlaschek, M., Tantcheva-Poor, I., Naderi, L., Ma, W., Schneider, L.A., Razi-Wolf, Z., et al. (2001) Solar UV Irradiation and Dermal Photoaging. Journal of Photochemistry and Photobiology B, 63, 41-51. http://dx.doi.org/10.1016/S1011-1344(01)00201-9

[8] Bernhard, D., Moser, C., Backovic, A. and Wick, G. (2007) Cigarette Smoke an Aging Accelerator? Experimental Gerontology, 42, 160-165. http://dx.doi.org/10.1016/j.exger.2006.09.016

[9] Yin, L., Morita, A. and Tsuji, T. (2001) Skin Aging Induced by Ultraviolet Exposure and Tobacco Smoking: Evidence from Epidemiological and Molecular Studies. Photodermatology Photoimmunology \& Photomedecine, 17, 178-183. http://dx.doi.org/10.1034/j.1600-0781.2001.170407.x

[10] Poljsak, B., Dahmane, R.G. and Godic, A. (2012) Intrinsic Skin Aging: The Role of Oxidative Stress. Acta Dermatovenerogica Alpina, Pannonicaet Adriatica, 21, 33-36.

[11] Hayes, B.T., Merrick, M.A., Sandrey, M.A. and Cordova, M.L. (2004) Three-MHz Ultrasound Heats Deeper into the Tissues than Originally Theorized. Journal of Athletic Training, 39, 230-234.

[12] Murray, A.K., Herrick, A.L., Gorodkin, R.E., Moore, T.L. and King, T.A. (2005) Possible Therapeutic Use of Vasodilator Iontophoresis. Microvascular Research, 69, 89-94. http://dx.doi.org/10.1016/j.mvr.2005.01.005

[13] Rubinsky, B. (2007) Irreversible Electroporation in Medicine. Technology in Cancer Research and Treatment, 6, 255260. http://dx.doi.org/10.1177/153303460700600401

[14] Wessling, K.C., DeVane, D.A. and Hylton, C.R. (1987) Effects of Static Stretch versus Static Stretch and Ultrasound Combined on Triceps Surae Muscle Extensibility in Healthy Women. Physical Therapy, 67, 674-679.

[15] Draper, D.O., Castel, J.C. and Castel, D. (1995) Rate of Temperature Increase in Human Muscle during 1 MHz and 3 MHz Continuous Ultrasound. Journal of Orthopaedic \& Sports Physical Therapy, 22, 142-150. http://dx.doi.org/10.2519/jospt.1995.22.4.142

[16] Rose, S., Draper, D.O., Schulthies, S.S. and Durrant, E. (1996) The Stretching Window, Part Two: Rate of Thermal Decay in Deep Muscle Following 1-MHz Ultrasound. Journal of Athletic Training, 31, 139-143.

[17] Neumann, E., Schaefer-Ridder, M., Wang, Y. and Hofschneider, P.H. (1982) Gene Transfer into Mouse Lyoma Cells by Electroporation in High Electrical Fields. The EMBO Journal, 1, 841-845.

[18] Napoli, C., Paolisso, G., Casamassimi, A., Al-Omran, M., Barbieri, M., Sommese, L., et al. (2013) Effects of Nitric Oxide on Cell Proliferation. Journal of the American College of Cardiology, 62, 89-95.

http://dx.doi.org/10.1016/j.jacc.2013.03.070 